\title{
Production and Applications of Radiopharmaceuticals: A Review
}

\author{
Nihar Ranjan Kar* \\ Centurion University of Technology and Management, IDCO Indrustrial Area, Pitamahal, Rayagada, Odisha, INDIA.
}

\begin{abstract}
Radiopharmaceuticals are exclusive medicinal formulations including radioisotopes which are utilized in foremost clinical areas for study, diagnosis and/or therapy. They are radioactive agents employed in nuclear medical field to demonstrate high and exact localization radioactivity into target tissue. Recently, however, there has been a considerable development of this division of nuclear medicine with the preamble of a quantity of new radionuclides and radiopharmaceuticals for the treatment of cancer, neuroendocrine disorder and other diseases. The application of definite radiotracers called radiopharmaceuticals for imaging organ function and disease states is an exceptional potential of nuclear medicine. Distinct other imaging modalities, for example- Computed Tomography (CT), Magnetic Resonance Imaging (MRI) and UItrasonography (US), nuclear medicine procedures are competent of drawing physiological function and metabolic activity and
\end{abstract}

thus giving more precise information about the organ function and dysfunction. This review focuses the production and therapeutic applications of radiopharmaceuticals which hold functional radio atoms.

Key words: Compounding of radiopharmaceuticals, Radionuclides, Radioactive decay, Radioactive emission diagnostic imaging, Nuclear medicine.

Correspondence

Nihar Ranjan Kar,

Centurion University of Technology and Management, IDCO Indrustrial Area, Pitamahal, Rayagada-765002, Odisha, INDIA.

Phone no: +91-9439511837

Email: nihar_795@rediffmail.com

DOI: 10.5330/ijpi.2019.2.8

\section{INTRODUCTION}

The grounds of molecular biology, immunology and genetics have produced numerous significant improvements that press on the perceptive of the introduction and development of oncological, cardiological and neurological ailments in addition to the detection of disease-related molecules and drugs that mainly target diseased cells all through the therapy. These imminent have generated the growth of targeted radiopharmaceuticals which launches a new aspects of radiopharmaceuticals in nuclear medicine. ${ }^{1}$

Radiopharmaceuticals have a short history compared to other drugs and medicinal products. The widespread use of radioactive nuclides for medical applications came as a direct result of the development of the atomic bomb during the Second World War. The construction of nuclear reactors in this context, opened up for the possibility of producing a whole range of new radionuclides by neutron activation of non-radioactive targets. Radionuclides that were not found in nature, could now be produced artificially in quantities that made it possible for use in scientific and medical applications. ${ }^{1}$ The 1990 's has seen major changes in the management of health care in many western countries. From being a largely unrestricted resource with open-ended budgets and complete clinical freedom, health-care provision has become highly controlled with established protocols, tight budgets and internal markets. Drug development has also become more tightly regulated. Radiopharmaceuticals now have to comply with the full range of regulations which control conventional drugs and this has enormously increased their development costs. The number of major commercial radiopharmaceutical players in the field is also decreasing following mergers and take-overs in the Industry and this limits the possibilities for commercial exploitation of academic discoveries. There have also been major upheavals in the institutions undertaking radiopharmaceutical development. ${ }^{2}$ Radiopharmaceuticals tend to differ from normal medicines in that they have a short half-life. Because of their rapid decay, they must be prepared shortly before their clinical use and comprehensive Quality Control (QC) of the final product is not possible: sterility testing, for instance, cannot be performed due to time limits. Safe and effective preparation and use of radiopharmaceuticals is, therefore, vital for the protection of the operator and the final user, the patient. The utilization of radioactive material requires cautious and secure usage of these products by trained and approved personnel, in consent/authorized laboratory facility as per the guidelines of Atomic Energy Regulatory Board (AERB) of India.

\section{Definitions and Terminology}

Radiopharmaceutical means any medicinal or pharmaceutical invention, which when ready for exercise includes one or more radionuclides (radioactive isotopes) meant for human application both for diagnosis or therapy.

Nuclide is an elemental species illustrated by its mass number 'A', (the sum of the number of protons and neutrons in its nucleus), its atomic number ' $Z$ ' (number of protons which is also same as number of electrons in a neutral atom) and also by its nuclear energy state.

Isotopes of an element are nuclides with the equal atomic number ' $\mathrm{Z}$ ' but dissimilar mass numbers ' $A$ '. They reside in the same position in the periodic table and contain analogous chemical properties.

Radionuclide: Nuclides having an unstable arrangement of protons and neutrons that alter unexpectedly to either a stable or another unstable mixture of protons and neutrons with a steady statistical possibility by releasing radiation. These are believed to be radioactive and are called radionuclides. The initial unstable nuclide is said as the 'parent radionuclide' and the nuclide after alteration as the 'daughter nuclide'. Such a conversion is also known as 'Radioactive transmutation' or 'radioactive disintegration' or 'radioactive decay'

Radioactivity: The occurrence of emission of radiation owing to the spontaneous transformation or disintegration of the radionuclide is 
known as 'Radioactivity'. However, the term radioactivity is also used to express the physical quantity (activity or strength) of this phenomenon. The radioactivity of a preparation is the number of nuclear disintegrations or transformations per unit time.

Units of Radioactivity: In the International System (SI), the unit of radioactivity is one nuclear transmutation per second and is expressed in Becquerel (Bq), named after the scientist Henri Bequerel. The old unit of radioactivity was Curie ( $\mathrm{Ci})$, named after the scientists Madame Marie Curie and Pierre Curie, the pioneers who studied the phenomenon of radioactivity. One $\mathrm{Ci}$ is the number of disintegrations emanating from $1 \mathrm{~g}$ of Radium-226 and is equal to 3.7 x $1010 \mathrm{~Bq}$. Absolute radioactivity measurements require a specialized laboratory, but identification and measurement of radiation can be carried out relatively by comparing with standardized preparations provided by Reference Laboratories recognized by the International or National Authorities. (With all statements involving radioactivity, it is necessary to include a reference date of measurement in case of radionuclides with half-life less than 30 days. The time of standardization should be expressed to the nearest hour. For radionuclides with a half-life period of less than one day, a more precise statement of reference time should be given.

Half-Life Period: The time in which a given quantity of a radionuclide decays to half its initial value is termed as half-life $\left(T_{1 / 2}\right)$.

Radionuclide generator: Any system or device incorporating a fixed parent radionuclide from which is produced a daughter radionuclide is extracted by elution or by any other method. For e.g., the most widely used radionuclide generator in radiopharmacy is $99 \mathrm{Mo}-99 \mathrm{mTc}$ generator.

Radionuclidic purity: The ratio, expressed as a percentage, of the radioactivity of the radionuclide of interest to the total radioactivity of the radioactive preparation is referred to as the 'Radionuclidic Purity'. In the context of radiopharmaceuticals, 'Radionuclidic purity' is an important quality parameter and it is mandatory that the radionuclidic impurities to be within the stipulated limits. Such radionuclidic impurities, arise during the radionuclide production, are hence, dependent on the production methodroute. In the context of radiopharmaceuticals, the acceptable limits for the possible radionuclides are listed in the individual monographs.

Isotopic carrier is a stable isotope of the element either present or added to the radioisotope of the same element. Often, the radionuclides contain isotopic carriers and their content depends on the route/method followed for the production of the radionuclide.

Radiochemical purity: The ratio, expressed as a percentage, of the radioactivity of the radionuclide of interest in a stated chemical form, to the total radioactivity of that radionuclide present in the preparation, is referred to as 'Radiochemical Purity'. In the context of radiopharmaceuticals, radiochemical purity is an important quality parameter, which needs to be within the stipulated limits. The relevant radiochemical impurities are listed with their limits in the individual monographs for each Radiopharmaceutical.

Chemical purity of a chemical substance is the percentage of the chemical of interest in the specified chemical form. In the monographs on radiopharmaceutical preparations, chemical purity of the active ingredient is indicated and controlled by specifying limits on chemical impurities.

Specific radioactivity: The radioactivity of a radionuclide per unit mass of the element or of the chemical form of the radioactive preparation is referred to as the 'Specific Radioactivity'; sometimes also referred as 'specific activity'.

Radioactive concentration: This refers to the radioactivity of a radionuclide per unit volume of the radioactive preparation.

Total radioactivity: The radioactivity of the radionuclide per unit of the dispensed formulation (vial, capsule, ampoule, generator, etc) is the total radioactivity, which is an important parameter in dispensing and administration of the radioactive material to the patient as well as from the regulatory requrement for safe handling of the radioactive materials in a facility.

Kit for radiopharmaceutical preparation: It is a set of non-radioactive reagents to be reconstituted and/or combined with radionuclides following the protocol suggested by the manufacturer for preparing the final radiopharmaceutical formulations, prior to its administration. Such kits are Also often referred to as 'cold kits', as they are devoid of radioactivity.

Radiopharmaceutical precursor: It is a chemical compound or ligand used in the synthesis of the radiopharmaceutical preparation. It could either be an inactive chemical compound or a radiolabeled intermediate produced for the preparation of radiopharmaceutical formulation, prior to administration.

Period of validity or Shelf life of Radiopharmaceutical: The time during which specifications described in the monograph are complied with, by the radiopharmaceutical denotes the shelf life of the radiopharmaceutical preparation. Any radiopharmaceutical preparations including the cold kits have limited shelf life, which needs to be clearly stated on the label as the expiry date and, if necessary, the time.

\section{Production of Radionuclides}

Radionuclides used in radiopharmaceuticals are produced artificially by the radioactive decay of other radioactive atoms. This production can be carried out by any of the following methods.

\section{Radionuclide Generator}

The radioisotope generator is an ion exchange column containing resin or alumina upon which a long-lived parent nuclide has been absorbed. Typical radionuclide generator is a glass or plastic column and the bottom of this is filled with adsorbent material on which parent nuclide is adsorbed. After the secular equilibrium that is after $4-5$ half lives, the daughter nuclide growth is eluted in carrier freestate with appropriate solvent. It includes production of ${ }^{68} \mathrm{Ga},{ }^{82} \mathrm{Rb},{ }^{99 \mathrm{~m}} \mathrm{Tc}$ and ${ }^{113 \mathrm{~m}} \mathrm{I}$ radionuclides, out of which ${ }^{99 \mathrm{~m}} \mathrm{Tc}$ is of particular importance. Due to its ideal imaging energy and physical half-life as well as the ability to bind to so many compounds, approximately $85 \%$ of all imaging procedures are performed following administrations of ${ }^{99 \mathrm{~m} T c}$. The versatile chemistry of technetium emerging from the 8 possible oxidation states, along with a proper understanding of structure-biologic activity relationship, has been exploited to yield a plethora of products meant for morphologic and functional imaging of different organs. Recent efforts have been

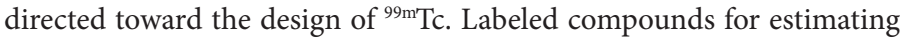
receptor or transporter functions. A number of bi-functional chelating agents that provide ${ }^{99 \mathrm{~m}} \mathrm{Tc}$ labeled proteins and peptides of high in vivo stability with high radiochemical yields have also been developed. ${ }^{3}$

\section{Cyclotron Produced Radionuclides}

The cyclotron and similar particle accelerators can be used only with charged particles such as electrons, protons and deuterons. This is because, the operation of such machines depend upon the interaction of magnetic and/or electrostatic fields with the change of particles undergoing acceleration. A beam of charged particles is produced by accelerating ions around a widening circle using magnetic field for control and electric current for acceleration. Various separation techniques are available to separate product from target. It is required that chemical forms of target and product must be different to effect separation. It includes production of positron emitting isotopes such as ${ }^{11} \mathrm{C},{ }^{13} \mathrm{~N},{ }^{15} \mathrm{O}$ and ${ }^{18} \mathrm{~F}$. Cyclotron yields are dependent upon number of target atoms, energy of particles, decay of product after it is formed, length of irradiation and isotope enrichment of target. ${ }^{3,4}$ 


\section{Cyclotron Produced}

An example of this method is production of ${ }^{22} \mathrm{Na}$, where a target of ${ }^{24} \mathrm{Mg}$ is bombarded with deuterons, i.e.

$$
{ }^{24} \mathrm{Mg}+{ }^{2} \mathrm{H} \quad{ }^{22} \mathrm{Na}+{ }^{4} \mathrm{He}
$$

A deuteron is the second most common isotope of hydrogen, i.e. ${ }^{2} \mathrm{H}$. When it collides with a ${ }^{24} \mathrm{Mg}$ nucleus, a ${ }^{22} \mathrm{Na}$ nucleus plus an alpha particle is produced. The target is exposed to the deuterons for a period of time and is subsequently processed chemically in order to separate out the ${ }^{22} \mathrm{Na}$ nuclei. ${ }^{5}$

\section{Pile Produced Isotope}

Most of the radioactive materials produced today for use in industry, academic research and medicine are produced in a nuclear pile (nuclear reactor). Uranium fission reaction produces a large supply of neutrons. One neutron for each uranium atom, undergoing fission is used to sustain the reaction. The remaining neutrons are used either to produce plutonium or used to produced radioactive products by causing the neutrons to interact with specific substances, which have been inserted into the pile, the latter process being known as neutron activation. Production of ${ }^{133} \mathrm{Xe},{ }^{99} \mathrm{Mo}$ and ${ }^{131} \mathrm{I}$ is carried out by this method. ${ }^{6}$

\section{Thermal Neutron Reactor-produced Radioisotope}

For thermal neutron reactor-produced radioisotopes, reactor is source of thermal neutrons. An (n, gamma) reaction occurs. It causes increase of atomic weight by one and no change in atomic number. Same element is therefore present. ${ }^{\text {? }}$

E.g. ${ }^{98} \mathrm{Mo}$ after reaction produces ${ }^{99} \mathrm{Mo}$.

\section{Commonly used Radiation Sources}

There are many radiation sources used in healthcare system. The differences relate to the position of the radiation source; external beam radiation therapy is outside the body, brachytherapy uses sealed radioactive sources placed precisely in the area under treatment and systemic radioisotopes are given by infusion or oral ingestion. ${ }^{8}$

\section{External Beam Radiotherapy}

The radiation is beamed into the tumor from outside the body for approximately two to three minutes for each treatment. External beam radiotherapy (EBRT) or teletherapy is the most common form of radiotherapy. The patient sits or lies on a couch and an external source of ionizing radiation is pointed at a particular part of the body. In contrast to internal radiotherapy (brachytherapy), in which the radiation source is inside the body, external beam radiotherapy directs the radiation at the tumour from outside the body. ${ }^{9}$

\section{Image-guided, Intensity-modulated Radiation Therapy}

Intensity-modulated Radiation Therapy (IMRT) is used to treat patients with prostate cancer, cancers of the head and neck and cancer at the base of the skull or parts of the brain. IMRT allows the tumor to be targeted with higher doses of radiation, particularly in the vicinity of critical structures, with greater potential for a cure and greater likelihood of limiting late complications from treatment. The incorporation of imageguided technology with IMRT allows doctors to track tumor position and location while the patient is actually on the treatment table. Imageguided IMRT affords more precise coverage, allowing doctors to respond immediately to any tumor movement and, if necessary, to recalculate the radiation fields during the treatment session. ${ }^{10}$ Stereostatic Radiosurgery A precise, single dose of radiation is delivered in cases where tumors of the brain are adjacent to critical areas, such as the brainstem, eyes or optic nerves. Stereotactic radiosurgery further minimizes the side effects associated with conventional radiation. A neurosurgeon is present during treatment to attach a halo-like frame to the scalp to assist with proper positioning. CyberKnife is a robotic system for stereotactic radiosurgery. ${ }^{11}$

\section{Stereostatic Radiotherapy}

Similar to stereostactic radiosurgery, lower doses of focused radiation are delivered with pinpoint accuracy during a series of treatment sessions that usually last between eight to 10 days. This method of multiple treatments is called "fractionation." During treatment, a halo-like frame held in place by a mouthpiece helps position the head for treatment. ${ }^{12}$

\section{Virtual Simulation and 3-Dimensional Radiation Therapy}

The planning of radiation therapy treatment has been revolutionized by the ability to delineate tumors and adjacent normal structures in three dimensions using specialized CT and/or MRI scanners and planning software.

Virtual simulation, the most basic form of planning, allows more accurate placement of radiation beams than is possible using conventional Xrays, where soft-tissue structures are often difficult to assess and normal tissues difficult to protect.

An enhancement of virtual simulation is 3-dimensional conformal radiation therapy (3DCRT), in which the profile of each radiation beam is shaped to fit the profile of the target from a Beam's Eye View (BEV) using a Multileaf Collimator (MLC) and a variable number of beams. ${ }^{12}$ When the treatment volume conforms to the shape of the tumor, the relative toxicity of radiation to the surrounding normal tissues is reduced, allowing a higher dose of radiation to be delivered to the tumor than conventional techniques would allow. ${ }^{13}$,

\section{Volumetric Modulated Arc Therapy (VMAT)}

Volumetric Modulated Arc Therapy (VMAT) is a new radiation technique, which can achieve highly conformal dose distributions on target volume coverage and sparing of normal tissues. The specificity of this technique is to modify the three parameters during the treatment. VMAT delivers radiation by rotating gantry (usually $360^{\circ}$ rotating fields with one or more arcs), changing speed and shape of the beam with a Multi-Leaf Collimator (MLC) ("sliding window" system of moving) and fluence output rate (dose rate) of the medical linear accelerator. VMAT also has the potential to give additional advantages in patient treatment, such as reduced delivery time of radiation, compared with conventional static field Intensity Modulated Radiotherapy (IMRT). ${ }^{14}$

\section{Particle Therapy}

In particle therapy (proton therapy being one example), energetic ionizing particles (protons or carbon ions) are directed at the target tumor. The dose increases while the particle penetrates the tissue, up to a maximum (the Bragg peak) that occurs near the end of the particle's range and it then drops to (almost) zero. The advantage of this energy deposition profile is that less energy is deposited into the healthy tissue surrounding the target tissue. ${ }^{15}$

\section{Auger Therapy}

Auger Therapy (AT) makes use of a very high dose of ionizing radiation in situ that provides molecular modifications at an atomic scale. AT differs from conventional radiation therapy in several aspects; it neither relies upon radioactive nuclei to cause cellular radiation damage at a cellular dimension, nor engages multiple external pencil-beams from different directions to zero-in to deliver a dose to the targeted area with reduced dose outside the targeted tissue/organ locations. Instead, the in-situ delivery of a very high dose at the molecular level using AT aims for 
in situ molecular modifications involving molecular breakages and molecular re-arrangements such as a change of stacking structures as well as cellular metabolic functions related to the said molecule structures. ${ }^{16}$

\section{Total Body Irradiation (TBI)}

Patients who are awaiting a bone-marrow or stem-cell transplant can receive this important preparatory treatment. When combined with chemotherapy, TBI is used to suppress the immune system and eliminate any pre-existing tumor cells. TBI treatments can last anywhere from 20 to $60 \mathrm{~min}$. Additional time may be needed for treatment planning and simulation. The specific amount of radiation the doctor prescribes is divided into a number of doses and may be given one or more times a day for one to four days, depending on the individual case. A specially designed stand may be used for support during treatment. The stand includes a bicycle seat and hand grips for support. Alternatively, a patient may be treated lying on a stretcher. ${ }^{17}$

\section{Prostate seed implants (Brachytherapy)}

An advanced form of radiation therapy, also called brachytherapy, uses radioactive seed implants to treat early prostate cancer. In this minimally invasive procedure, doctors use ultrasound-guided needles to insert tiny radioactive seeds (Iodine 125 or Palladium 103) into the prostate gland. The seeds emit low-energy gamma rays that specifically target cancer cells in the prostate. The seeds lose 90 percent of their radioactivity within a few months following the implant and within a year they are considered inert or not active. ${ }^{18}$

\section{Deep inspiration breath-hold}

Deep Inspiration Breath-hold (DIBH) is a method of delivering radiotherapy while limiting radiation exposure to the heart and lungs. It is used primarily for treating left-sided breast cancer. The technique involves a patient holding their breath during treatment. There are two basic methods of performing DIBH: free-breathing breath-hold and spirometry-monitored deep inspiration breath hold. ${ }^{19}$

\section{Diagnostic Imaging Technique CT (Computer tomography)}

The principal of $\mathrm{CT}$ is the measuring of the spatial distribution of physical material to be examined from different directions and to compute superposition free images from this data. It is basically a technique of X-ray photography by which a single plane of a patient is scanned from various angles in order to provide a cross-sectional image of the internal structure of that plane. A CT scan has many diagnostic clinical applications. It improves the diagnosis accuracy by delineating details of the organs including soft tissues and bones. CT scan can provide information about the spread of an infection or tumors to different body parts and can assist surgical interventions, biopsies and radiotherapies. ${ }^{20}$

\section{MRI (Magnetic Resonance Imaging)}

An MRI is similar to a Computerized Topography (CT) scanner in that it produces cross-sectional images of the body. Looking at images of the body in cross section can be compared to looking at the inside of a loaf of bread by slicing it. Unlike a CT scan, MRI does not use X-rays. Instead, it uses a strong magnetic field and radio waves to produce very clear and detailed computerized images of the inside of the body. MRI is commonly used to examine the brain, spine, joints, abdomen and pelvis. A special kind of MRI exam, called Magnetic Resonance Angiography (MRA), examines the blood vessel. ${ }^{21}$

\section{Echography/Ultrasound}

Ultrasound uses high-frequency sound waves to look at organs and structures inside the body. Health care professionals use them to view the heart, blood vessels, kidneys, liver and other organs. During pregnancy, doctors use ultrasound tests to examine the fetus. Unlike x-rays, ultrasound does not involve exposure to radiation. During an ultrasound test, a special technician or doctor moves a device called a transducer over part of your body. The transducer sends out sound waves, which bounce off the tissues inside your body. The transducer also captures the waves that bounce back. Images are created from these sound waves. ${ }^{22}$

\section{Nuclear Medicine}

Nuclear medicine is a mainly medical diagnostic discipline for imaging metabolism and other functional processes in the human body. Prior to the imaging process a radioactively labeled tracer is administered to the patient. The strength of the technique lies in the fact that substances move to organ systems in very selective ways. Labeling these substances to radioactive tracers (particularly technetium) enables imaging of the distribution of such substances in the human body with the aid of gamma cameras or PET scanners. Three different modalities are available for this process: planar scintigraphy, SPECT (single photon emission computed tomography) and PET (positron emission tomography). ${ }^{23}$

\section{Planar Scintigraphy}

It is the simplest available technique, yielding a two-dimensional projection image of tracer activity distribution in the human body. The technique is based on gamma radiation that is created in the decay process of a radionuclide. ${ }^{24}$

\section{SPECT (Single-photon Emission Computed Tomography)}

It was developed on the basis of planar imaging, which involves gamma cameras taking series of planar shots during rotations around the patient. It generates three dimensional images of nuclear activity distribution, enabling the physician to view activity distributions in cross-sections of the human body. ${ }^{25}$

\section{PET (Positron Emission Tomography)}

It has entered clinical practice in the last few decades. PET is an imaging technique whereby a radioactive isotope (a PET radionuclide) is administered into the patient's body. During decay, the isotope produces positrons (particles with the mass of electrons but with a positive charge). Electron and positron interaction causes the annihilation of both particles, releasing energy in the form of two gamma photons. The resulting gamma rays are detected by a ring of hundreds of detectors. ${ }^{26}$

\section{Intra-cavitary Therapy}

Direct intra-cavitary administration is a means of deliver in radiopharmaceuticals in high concentration to tumors that are spread out over the serosal linings of cavities and tumors cell presents in the malignant effusions. In order to minimize leakage of the radionuclide from the cavity, it is usually given in the form of radio colloid. Intracavitary therapy is applied to the peritoneal, pleural and pericardial cavities as well as to cystic brain tumors and to the spinal canal. Colloidal ${ }^{198} \mathrm{Au}$ was formerly the most widely used agent, but the radionuclide emits unwanted gamma radiation, leading to unnecessary exposure of non-target tissue within the patient. The agents of choice are now ${ }^{32} \mathrm{P}$ and ${ }^{90} \mathrm{Y}$ radio colloids, with perhaps radio labeled antibodies having a wider role to apply in the future. $^{27}$ 


\section{Imagining Status}

In recent years, scanning techniques have developed rapidly and are now among the most useful tools in diagnostic medicines. By means of scanning, tissues and organs can be visualized and such visualization facilitates the detection of abnormalities in their function. Radioactive materials are administered to the individual and distribution of the radioactive material in the body is measured by using imaging techniques. ${ }^{28}$

\section{Cardiovascular Imaging}

Radiopharmaceuticals are useful in cardiac imaging as agents that provide information of regional myocardial blood perfusion. There administered to provide information at peak cardiac output. The study involves stressing the patient with exercise in a treadmill or giving an i.v. injection of dipyridamole. An injection of thallium chloride or technetium-99m ( ${ }^{99 \mathrm{~m} T c}$ ) labeled methoxy isobutyl isonitrile is then given an imaging is carried out. ${ }^{29}$

\section{Bone Imaging}

This method widely used in the diagnosis of benign and malignant, primary and metastatic bone tumors. Bone imaging radiopharmaceuticals consist of diagnostic (primarily single photon emitters) and therapeutic agents. The therapeutic radiopharmaceuticals are utilized on the basis of their particulate emissions (primarily Beta) and those are treated differently than the single photon bone imaging agents. For the in vivo studies, ${ }^{99 \mathrm{~m}} \mathrm{Tc}$ labeled polyclonal human immunoglobulin $\left({ }^{99 \mathrm{~m}} \mathrm{Tc}-\mathrm{HIG}\right)$ was used as the radiopharmaceutical to demonstrate arthritic lesions by gamma scintigraphy. After the induction of arthritis in knee joints of rabbits, the radio-labeled microspheres loaded with DS were injected directly into the articular cavity and gamma scintigrams were obtained at periodic intervals to find the residence time of microspheres in the knee joints in order to determine the most suitable formulation. ${ }^{30}$

\section{Lung Imaging}

The main purpose of the long imaging is the diagnosis of pulmonary emboli and to evaluate pulmonary perfusion and pulmonary ventilation and to assess pulmonary function prior to pneumonectomy. The agent used is ${ }^{99 \mathrm{~m}} \mathrm{Tc}$ macro aggregated albumin. Iseri et al. prepared albumin and gelatin microsphere incorporating a tuberculostatic agent, rifampicin and its in vivo distribution was studied by causing its accumulation in the target organ, i.e., lung Bio-distribution was determined by intravenous administration of particles if $25-27$ microns ${ }^{99 \mathrm{~m}} \mathrm{Tc}$ - labeled microspheres to Swiss mice. The radioactivity of lungs was compared with that of the liver, spleen, kidney, stomach and heart. The percentage accumulation was higher in the lungs than in the other organs for both albumin and gelatin microspheres. ${ }^{31}$

\section{Renal Imaging}

This is used to determine renal function, renal vascular flow and renal morphology. These are also used for evaluation of renal transplant patients for complication such as obstruction, infarction, leakage, tubular, necrosis and rejection. ${ }^{99 \mathrm{~m}} \mathrm{Tc}$-diethylenetriaminepentaacitic acid and ${ }^{131} \mathrm{I}$-ibdohippurate are commonly used radiopharmaceuticals. ${ }^{32}$

\section{Spleen Imaging}

Radiopharmaceuticals are used for spleen imaging for various purposes. Spleen imaging is performed by using ${ }^{99 \mathrm{~m}} \mathrm{Tc}$-denatured erythrocytes. After a blood sample is withdrawn from the patient, the erythrocytes are labeled with ${ }^{99 \mathrm{~m} T c}$ in vitro; the labeled cells are denatured by heating at $49.5^{\circ} \mathrm{C}$ for $15 \mathrm{~min}$ following reinjection into the patient, the cells are then taken of by the spleen. Imaging with ${ }^{99 \mathrm{~m} T c-d e n a t u r e d ~ e r y t h r o c y t e s ~}$ is used to detect spleen nucleus. ${ }^{33}$

\section{Imaging of Inflammatory Lesions}

Scintigraphic imaging of inflectional inflammation is a powerful diagnostic tool in the management of patients with infectious or inflammatory diseases. Many radiopharmaceuticals have been introduced for the scintigraphic demonstration of infectious and inflammatory lesions. They can be classified into two major categories according to their specificity. Specific radiopharmaceuticals include in vitro labeled leukocytes, radio-labeled monoclonal antibodies and receptor specific small proteins and peptides. Non-specific radiopharmaceuticals include, radio-labeled nanocolloids, liposomes, macro molecules such as human immunoglobulin, dextran and human serum albumin..$^{34}$

\section{Gastrointestinal Imaging}

Latex particles coated with either amino or carboxyl group can be efficiently labeled with ${ }^{99 \mathrm{~m}} \mathrm{Tc}$ and used in the studies of gastrointestinal function. Ercan et al. prepared ${ }^{99 \mathrm{~m}} \mathrm{Tc}$-labeled mono-disperse latex particles coated with amino or carboxyl groups for studies of GI function. ${ }^{35}$

\section{Brain Imaging}

Brain imaging is performed using radiopharmaceuticals by single photon emission computed tomography (SPECT) and positron emission tomography (PET). SPECT and PET radiopharmaceuticals are classified according to blood-brain-barrier (BBB) permeability, cerebral perfusion and metabolism receptor-binding and antigen antibody binding. The blood brain barrier SPECT agents, such as ${ }^{99 \mathrm{~m} T c-D T P A},{ }^{201} \mathrm{Ti}$ and ${ }^{67} \mathrm{Ga}$-citrated are excluded by normal brain cells but enter into tumor cell because of altered BBB. The radiopharmaceuticals uptake into tumor cells was rapid and temperature and $\mathrm{pH}$-dependent. The radioactivity concentration in tumor cells varied from 10 to $33 \%$ of the total activity following $30 \mathrm{~min}$ incubation at $37^{\circ}$ ( $\mathrm{pH}$ 7.4). In comparison, accumulation of the radiopharmaceuticals into normal brain and pancreatic tissue remained relatively low. Imaging of brain tumors require a disrupted $\mathrm{BBB}$, however, it is intact in the early stages of brain tumor growth, when diagnosis is most critical. Relative to normal brain, brain tumor cells frequently over express peptide receptors, such as the receptor for epidermal growth factor (EGF). Peptide radiopharmaceuticals such as radio labeled EGF could be used to image early brain tumors. ${ }^{36}$

\section{Optical Imaging}

Optical imaging is a technique based on interference and the bending of light that is fired onto a body or tissue from a laser or infrared light source. The body is injected with proteins marked by, for example, a fluorescent marker. Optical imaging may be subdivided into diffusion and ballistic imaging systems. Since the penetrability of the body in relation to light is relatively small, optical imaging is unsuitable in certain contexts, for example organ examinations. ${ }^{37}$

\section{Therapeutic Applications of Radiopharmaceuticals treatment of Hyperthyrodism}

131-iodine is used extensively for the treatment of hyperthyroidism. Upon oral administration of the radionuclide, approximately $60 \%$ of the radioactivity is taken up by the overactive gland. The principal disadvantage of the radioiodine therapy is the high incidence of early and late hypothyroidism, making it necessary to monitor patients adequately after treatment. As a consequence, treatment is no longer restricted to older patients and now adolescencents and even children, being treated with radio iodine. ${ }^{38}$ 


\section{Treatment of Thyroid Carcinoma}

Radioiodine has been used for several decades in the treatment of differentiated thyroid carcinoma, a tumor which metastases to bone, lungs and other soft tissues. However, it is sloe growing and the prognosis is relatively good, allowing long term follow up treated patients. Repeating radionuclide imaging with radioiodine can assess response to therapy and, if necessary, further therapeutic dosages of ${ }^{131} \mathrm{I}$ may be required in advanced or resistant cases. ${ }^{39}$

\section{Treatment of Neuroendocrine Tumors}

Metaiodobenzyleguanidine is similar in structure to the adrenergic neuron blocker guanethidine and the neurotransmitter nor-adrenaline. Due to the structural similarity, it is taken of by the adrenal medulla and other tissues with rich sympathetic innervations but unlike nor-adrenaline it is not metabolized and is largely excreted unchanged in the urine. ${ }^{131} \mathrm{I}$-metaiodobenzyleguanidine $\left({ }^{131} \mathrm{I}-\mathrm{MIBG}\right.$ ) has been used with success in the treatment of neuroendocrine tumors such as neuroblastoma, carcinoid tumors and meduliary carcinoma of the thyroid. Several other agents are being investigated as alternatives to ${ }^{131} \mathrm{I}-\mathrm{MIBG}$ and include 161-Terbium-diethylenetriaminepentaacetic acid-octreotide and 111-Indium-diehylnetriaminepentaacetic acid ( ${ }^{111}$ In-DTPA) for tumors containing somatostatin receptors. ${ }^{40}$

\section{Treatment of Bone Tumors}

Bone metastases are a common finding in patients suffering from cancers of the prostate, breast and lungs and in these patients control of bone pain is a significant clinical problem. External beam radiotherapy, in combination with analgesic drugs, remains the mainstay of treatment but the proportion of the body that can be treated is limited. Several beta emitting radionuclides, in a variety of chemical forms, can be used for the treatment of bone metastases. ${ }^{32} \mathrm{P},{ }^{89} \mathrm{Sr}, 186$-Rhenium and 153-Samaraum have been carried out. ${ }^{41}$

\section{Treatment of Myeloeproliferative Diseases}

${ }^{32} \mathrm{P}$ has been used for more than 50 years in the treatment of a variety of haematological disorders. Following intravenous injection, ${ }^{32} \mathrm{P}$ as the orthophosphate, is concentrated selectively by rapidly proliferating tissue and there is also bone uptake. In this way, a significant radiation dose is delivered to the bone narrow and results in growth retardation of the haemopoetic cell lines. The primary application of ${ }^{32} \mathrm{P}$ is in the treatment of polycythaemia rubra vera. In this condition there is an abnormal increase in the number of red cells in the circulation. However, treatment with phlebotomy, radioactive phosphorus or chemotherapy all results in significant increase in life expectancy. ${ }^{42}$

\section{Advantages of Radiopharmaceuticals in Healthcare System}

- It can be used as diagnosis and treatment of patient.

- It can provide fast onset of pain relief.

- It is common to cure cancer.

- Can treat multiple disease sites.

- Widely available mode of treatment.

- Directly treats tumor, especially useful for bone metastasis.

- Single dose is effective for some patients.

- Nuclear medicine tests can be performed on children.

- Nuclear medicine procedures have no side effects and are completely safe. ${ }^{42}$
Disadvantages of Radiopharmaceuticals in Healthcare

System

- When multiple fractions are given, it may produce prolonged inconvenience and discomfort for patients.

- Higher doses of head and neck radiation can be associated with cardiovascular complication, thyroid dysfunction and pituitary axis dysfunction.

- Nuclear medicine tests are non-recommended for pregnant women, because unborn babies have a greater sensitivity to radiation than children or adults.

- $\quad$ Filling in patient's teeth, dental braces and permanent bridges may cause some distortion around the mouth area.

- Can produce some allergic reactions.

- It has a radiation risk.

- Myelosupression may occur, especially with prior chemotherapy. ${ }^{43}$

\section{CONCLUSION}

Nowadays there are different types of radiopharmaceuticals are available and having an important role in diagnosis of disease. Recently, however, there has been a significant growth of this branch of nuclear medicine with the introduction of a number of new radionuclides and radiopharmaceuticals for the treatment of metastatic bone pain, neuroendocrine and other tumors. Today the field of radionuclide therapy is going through an extremely interesting and exciting phase and is poised for greater growth and development in the coming years.

\section{ACKNOWLEDGEMENT}

The author is thankful to Prof.(Dr.) S.C. Dinda, Professor of Pharmaceutics, College of Pharmacy, Teerthanker Mahaveer University, Delhi Road, Moradabad (U.P.) India-244001 for providing necessary information to frame the article.

\section{CONFLICT OF INTEREST}

The authors declare no conflict of interest.

\section{REFERENCES}

1. Markus M, Wolfgang W. Imaging Biomarkers or Biomarker Imaging?. Pharmaceuticals. 2014;7(7):765-78. doi:10.3390/ph7070765

2. Venugopal V. Societal Application of Nuclear Technology in health care, Industry and Water Resource Management in India. Energy Procedia. 2011;7:553-9.

3. Reetesh M, Richa B, Sonam J. A Review on Therapeutic Approach of Radiopharmaceutical in Health Care System. International Journal of Pharmaceutical and Biological Archives. 2012;3(3):487-92.

4. Frank R. Radiochemistry and Radiopharmaceutical Chemistry for Medicine Radiochemistry and Nuclear Chemistry: Encyclopedia of life support systems and UNESCO. 2010;2:63-94.

5. Chourasia MK, Ashawat MS, Neeti J, Chalasani KB, Jain RK, Sabitha M, et al. Radiopharmaceuticals: Preparation, Evaluation and Application, Indian. J Pharm Science. 2003;65(5):439-49.

6. Vera-Rulz H. Radiopharmaceuticals as therapeutic agents in Medical care and Treatment: A new IAEA research programme assists countries interested in the use of improved radiopharmaceuticals for medical therapy. IAEA Bulletin. 1993;35(1):24-7.

7. Sudipta C, Vimalnath KV, Rajeswari A, Ajit S, Sharma HD, Kamaleswaran K, et al. Preparation, Evaluation and first clinical use of 177Lu - Labeled hydroxyapatite particles in the treatment of Rheumatoid arthritis: Utility of cold kits for convenient dose formulation at hospital radio-pharmacy. J Label Compd Radiopharm. 2014;57(7):453-62.

8. Reetesh M, Richa B, Sonam J. A Review on Therapeutic Approach of Radiopharmaceutical in Health Care System. Int J Pharma and Bio Archi. 2012;3(3):487-92.

9. Wynn AV, Timothy JH. Therapeutic Radiopharmaceuticals. Chem Rev. 1999;99(9):2269-92

10. Maecke HR, Reubi JC. Somatostatin receptors as targets for nuclear medicine imaging and radionuclide treatment. J Nucl Med. 2011;52(6):841-4

11. Graham MM. Clinical molecular imaging with radiotracers: Current status. Med 
Princ Pract. 2012;21(3):197-208.

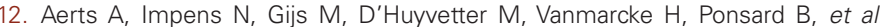
Biological Carrier Molecules of Radiopharmaceuticals for Molecular Cancer Imaging and Targeted Cancer Therapy. Curr Pharm Des. 2014;20(32):5218-44.

13. Asabella AN, Cascini GL, Altini C, Paparella D, Notaristefano A, Rubini G, et al. The copper radioisotopes: A systematic review with special interest to $64 \mathrm{Cu}$. Biomed Res Int. 2014;786463.

14. Attard AR, Chappell MJ, Bradwell AR. Model based calculation for effective cancer radioimmunotherapy. Br J Radiol. 1995;68(810):636-45.

15. Baczyk M, Czepczynski R, Milecki P, Pisarek M, Oleksa R, Sowinski J. 89Sr versus 153Sm-EDTMP: Comparison of treatment efficacy of painful bone metastases in prostate and breast carcinoma. Nucl Med Commun. 2007:28(4):245-50.

16. Baidoo KE, Yong K, Brechbiel MW. Molecular pathways: Targeted alpha-particle radiation therapy. Clin Cancer Res. 2013;19(3):530-7.

17. Barbet J, Bardies $M$, Bourgeois $M$, Chatal JF, Cherel M, Davodeau F, et al. Radiolabeled antibodies for cancer imaging and therapy. Methods Mol Biol. 2012;907:681-97.

18. Bayouth JE, Macey DJ, Kasi LP, Garlich JR, McMillan K, Dimopoulos MA, et al. Pharmacokinetics, dosimetry and toxicity of holmium-166-DOTMP for bone marrow ablation in multiple myeloma. J Nucl Med. 1995;36(5):730-7.

19. Blumgart HL, Yens OC. Studies on the velocity of blood flow: I. The method utilized. J Clin Invest. 1927:4(1):1-13.

20. Bodei L, Handkiewicz-Junak D, Grana C, Mazzetta C, Rocca P, Bartolomei M, et al. Receptor radionuclide therapy with 90Y-DOTATOC in patients with medullary thyroid carcinomas. Cancer Biother Radiopharm. 2004;19(1):65-71.

21. Bodei L, Mueller-Brand J, Baum RP, Pavel ME, Horsch D, O'Dorisio MS, et al. The joint IAEA, EANM and SNMMI practical guidance on peptide receptor radionuclide therapy (PRRNT) in neuroendocrine tumours. Eur J Nucl Med Mol Imaging. 2013;40(5):800-16

22. Bolzati C, Boschi A, Uccelli L, Tisato F, Refosco F, Cagnolini A, et al. Chemistry of the strong electrophilic metal fragment $[(99) T c(N)(P X P)](2+)(P X P=$ diphosphine ligand): A novel tool for the selective labeling of small molecules. J Am Chem Soc. 2002;124(38):11468-79.

23. Boschi A, Bolzati C, Uccelli L, Duatti A, Benini E, Refosco F, et al. A class of asymmetrical nitrido $99 \mathrm{mTc}$ heterocomplexes as heart imaging agents with improved biological properties. Nucl Med Commun. 2002;23(7):689-93

24. Boschi A, Uccelli L, Bolzati C, Duatti A, Sabba N, Moretti E, et al. Synthesis and biologic evaluation of monocationic asymmetric $99 \mathrm{mTc}$-nitride heterocomplexes showing high heart uptake and improved imaging properties. J Nucl Med. 2003;44(5):806-14

25. Boyd GS, Merrick MV, Monks R, Thomas IL. Se-75 Labeled bile acid analogs, new radiopharmaceuticals for investigating the enterohepatic circulation. J Nucl Med. 1981;22(8):720-5

26. Brans B, Bodei L, Giammarile F, Linden O, Luster M, Oyen WJ, et al. Clinical radionuclide therapy dosimetry: The quest for the "Holy Gray". Eur J Nucl Med Mol Imaging. 2007;34(5):772-86.

27. Bristow MR, Ginsburg R, Minobe W, Cubicciotti RS, Sageman WS, Lurie K, et al. Decreased catecholamine sensitivity and betaadrenergic-receptor density in failing human hearts. N Engl J Med. 1982;307(4):205-11.

28. Buchmann I, Meyer RG, Mier W, Haberkorn U. (2009) Myeloablative radioim- munotherapy in conditioning prior to haematological stem cell transplantation: Closing the gap between benefit and toxicity?. Eur J Nucl Med Mol Imaging. 2009;36(3):484-98.

29. Cornelissen B, Darbar S, Kersemans V, Allen D, Falzone N, Barbeau J, et al. Amplification of DNA damage by a gammaH2AX-targeted radiopharmaceutical. Nucl Med Biol. 2012;39(8):1142-51.

30. Denis-Bacelar AM, Romanchikova M, Chittenden S, Saran FH, Mandeville $\mathrm{H}$, Du $Y$, et al. Patient-specific dosimetry for intracavitary 32P-chromic phosphate colloid therapy of cystic brain tumours. Eur J Nucl Med Mol Imaging. 2013;40(10):1532-41.

31. Czisch M, Wehrle R, Kaufmann $C$, Wetter TC, Holsboer F, Pollmacher T, et al. Functional MRI during sleep: BOLD signal decreases and their electrophysiological correlates. Eur J Neurosci. 2004;20(2):566-74.

32. Umprayn K, Chitropas P, Amarekajorn S. Development of terbutaline sulphate sustained release coated pellets. Drug Dev Ind Pharm. 1999;25(4):477-91.

33. Claudio N, Rita C, Elisabetta E, Alberto G, Alessandro S, Carlo V, et al. Influence of the formulation and process parameters on pellet production by powder layering technique. AAPS Pharm SciTech. 2000;1(2):1-12.

34. Handa AK, Kerudi AV, Bhalla HL. Development and evaluation of ethylcellulose coated controlled release pellets. Ind J Pharm Sci. 2000;62(2):147-53.

35. Newton A, Podczeck F, Alderborn G, Chopra R. The influence of pellet shape and surface properties on the drug release from uncoated and coated pellets. Int $J$ Pharm. 2002;239(1):171-8

36. Zezhi JS, Moralesi L, Diaz S, Muhammadi NA. Drug release from kollicoat SR $30 \mathrm{D}$ coated nonpareil beads: evaluation of coating level, plasticizer type and curing condition. AAPS Pharm SciTech. 2002;3(2):1-9.

37. Boldmeier R, Pearnchob N. Coating of pellets with micronized ethylcellulose particles by a dry powder coating technique. Int J Pharm. 2003;268(1-2):1-11

38. Weijia Z, Mcginity J. Influence of Eudragit ${ }^{\circledR}$ NE 30 D blended with Eudragit $\circledast$ $\mathrm{L} 30 \mathrm{D}-55$ on the release of phenylpropanolamine hydrochloride from coated pellets. Drug Dev Ind Pharm. 2003;29(3):357-66.

39. Rahman N, Yuen KH, Woei WJ. Formulation and evaluation of controlled release diltiazem pellets using Eudragit NE40. Acta Pharmaceutica Turcica. 2005;47(3):199-207.

40. Shivkumar HN, Desai BG, Sarasija S. Design and evaluation of pH sensitive multi-particulate systems for chronotherapeutic delivery of diltiazem hydrochloride. Ind J Pharm Sci. 2006;68(6):781-7.

41. Bendas ER, Ayres JW. Leaky enteric coating on ranitidine hydrochloride beads: Dissolution and prediction of plasma data. Eur J Pharm and Biopharmaceutics. 2008;69(3):977-85.

42. Wei J, Mingfeng Q, Xia S, Yungping Q, Mingming S. Preparation of slow release pellets. Advances in Therapy. 2004;21(4):238-48.

43. Heng PW, Wang L, Tang E, Liew CV. Drying efficiency and particle movement in coating-impact on particle agglomeration and yield. Int J Pharm. 2008;350(12):172-80.

Cite this article: Kar NR. Production and Applications of Radiopharmaceuticals: A Review. Int. J. Pharm. Investigation. 2019;9(2):36-42. 